\title{
Whole-Exome Sequencing in Nine Monozygotic Discordant Twins
}

\author{
Rong Zhang, ${ }^{*, 1}$ Holger Thiele, ${ }^{*, 2}$ Peter Bartmann, ${ }^{3}$ Alina C. Hilger, ${ }^{1}$ Christoph Berg, ${ }^{4,5}$ Ulrike Herberg, ${ }^{6}$ \\ Dietrich Klingmüller, ${ }^{7}$ Peter Nürnberg, ${ }^{2}$ Michael Ludwig, ${ }^{8}$ and Heiko Reutter ${ }^{1,3}$ \\ ${ }^{1}$ Institute of Human Genetics, University of Bonn, Bonn, Germany \\ ${ }^{2}$ Cologne Center for Genomics, University of Cologne, Cologne, Germany \\ ${ }^{3}$ Department of Neonatology, University of Bonn, Bonn, Germany \\ ${ }^{4}$ Department of Obstetrics and Prenatal Medicine, University of Bonn, Bonn, Germany \\ ${ }^{5}$ Department of Obstetrics and Prenatal Medicine, University of Cologne, Cologne, Germany \\ ${ }^{6}$ Department of Pediatric Cardiology, University of Bonn, Bonn, Germany \\ ${ }^{7}$ Division of Endocrinology and Diabetes, Department of Medicine I, University of Bonn, Bonn, Germany \\ ${ }^{8}$ Department of Clinical Chemistry and Clinical Pharmacology, University of Bonn, Bonn, Germany
}

By definition, monozygotic (MZ) twins carry an identical set of genetic information. The observation of early post-twinning mutational events was shown to cause phenotypic discordance among MZ twin pairs. These mutational events comprise genomic alterations at different scales, ranging from single nucleotide changes to larger copy-number variations (CNVs) of varying sizes, as well as epigenetic changes. Here, we performed whole-exome sequencing (WES) in nine discordant MZ twins to identify somatic mutational events in the affected twin that might exert a dominant negative effect. Five of these MZ twin pairs were discordant for congenital heart defects (CHD), two for endocrine disorders, one for omphalocele, and one for congenital diaphragmatic hernia (CDH). Analysis of WES data from all nine MZ twin pairs using the de novo probability tool DeNovoGear detected only one apparent de novo variation in TMPRSS13 in one of the CHD-affected twins. Analysis of WES data from all nine MZ twin pairs by using standard filter criteria without the de novo probability tool DeNovoGear revealed a total of 6,657 variations in which both the twin pairs differed. After filtering for variations only present in the affected twins and absent in in-house controls, 722 variations remained. Visual inspection for read quality decreased this number to 12 , present only in the affected twin. However, Sanger sequencing of the overall 13 variations failed to confirm the variation in the affected twin. These results suggest that somatic mutational events in coding regions do not seem to play a major role in the phenotypic expression of MZ discordant twin pairs.

Keywords: discordance, monozygotic, twin pairs, whole-exome sequencing

MZ twins develop from a single fertilized egg and thus are thought to carry an identical set of genetic information. However, early post-zygotic mutational events have been found to constitute a low but substantial proportion of de novo mutations in humans (Dal et al., 2014). These comprise genomic alterations at different scales, ranging from changes affecting only single nucleotides to larger CNVs of varying size as well as epigenetic differences. As outlined in a comprehensive review provided by Van Dongen et al. (2012), twin studies represent a powerful approach to gain insight into the genetic etiology underlying complex traits and trait variation. Also, extensive intra-individual genetic variations have been observed in human tissues (O'Huallachain et al., 2012; 2013) and CNVs in MZ twins also revealed somatic mosaicism in tissues arising from the same zygote (Bruder et al., 2008). Hence, the search for dif- ferences in the genetic constitution within discordant MZ twins has been suggested a promising approach in gene finding (Zwijnenburg et al., 2010). This assumption has been supported by observations that very early post-twinning mutational or epigenetic events can cause phenotypic discordance in MZ twins (Gervin et al., 2012; Helderman-van den Enden et al., 1999; Kondo et al., 2002; Kruyer et al.,

RECEIVED 19 August 2015; ACCEPTED 3 November 2015. First published online 18 December 2015.

ADDRESS FOR CORRESPONDENCE: Heiko Reutter, Department of Neonatology and Pediatric Intensive Care \& Institute of Human Genetics, University of Bonn, Sigmund-Freud-Str. 25, D-53127 Bonn, Germany. E-mail: reutter@uni-bonn.de

* These authors contributed equally to the manuscript. 
1994; Mitchell et al., 2011; Selmi et al., 2014; Taylor et al., 2008; Zwijnenburg et al., 2010).

In accordance with these observations, Breckpot et al. (2012) identified three possibly disease-causing de novo $\mathrm{CNV}$ s in one affected twin out of six MZ twin pairs discordant for CHD, and Kondo et al. (2002) identified a nonsense mutation in IRF6 in the affected twin only in MZ discordant twins for Van der Woude syndrome. Moreover, a posttwinning $\sim 1.3 \mathrm{Mb}$ de novo deletion had been identified in a concordant-affected twin pair with attention problems (AP; Ehli et al., 2012) that might be of value to explain the higher AP score in the twin showing the deletion.

To identify the cause of disease in this study, we performed WES in nine MZ twin pairs. These twin pairs were discordant for CHD, endocrine disorders, omphalocele, and congenital $\mathrm{CDH}$. We hypothesized that the affected twin would show a causative de novo variation.

\section{Materials and Methods Patients and DNA Isolation}

In our study, we included nine MZ twin pairs with different discordances who were recruited through the Department of Neonatology, Children's Hospital, Department of Obstetrics and Prenatal Medicine, Department of Medicine I, and through the Department of Pediatric Cardiology, University of Bonn. For all twins, parental DNA could be obtained. Written informed consent was obtained from all patients and/or the parents prior to study entry. The Ethics Committee of the Medical Faculty of the University of Bonn approved the study. Isolation of genomic DNA from blood was carried out by using the Chemagic DNA Blood Kit special (Chemagen, Baesweiler, Germany), while genomic DNA from saliva samples was isolated with the Oragene DNA Kit (DNA Genotek Inc., Kanata, Canada).

\section{Twin Pairs}

The phenotypic features of each affected twin and family data are listed in Table 1. All twin pairs were born to nonconsanguineous parents. There was an unremarkable family history in all cases. Because MZ twins share all their DNA sequence, while DZ twins share, on average, only one half, the zygosity of the twin pairs was confirmed by comparing the SNVs of each co-twin in a twin pair (99.8\% similar).

\section{Whole Exome Sequencing (WES) and Data Analysis}

Exonic and adjacent intronic sequences were enriched from genomic DNA using the NimbleGen SeqCap EZ Human Exome Library v2.0 enrichment kit. WES was performed using a 100bp paired-end read protocol due to the manufacturer's recommendations on an Illumina HiSeq2000 sequencer by the Cologne Center for Genomics (CCG), Cologne, Germany. On average, the sequence output reached 9.3 sequenced gigabases, from which $12 \%$ were marked as duplicates (Picard software), and the remaining $91 \%$ of reads could be mapped on the human hg19 reference genome (bwa-aln software). The mean coverage of targeted regions was 77-123 (on average, 95) and the percentage of targets that covered at least 30x ranged from 87 to $93 \%$ (on average, 90\%). The UnifiedGenotyper (GATK) and Mpileup (Samtools) software were used to call variations and the CCG software FUNC (unpublished) was used to annotate variations for functional consequences in respect of altered protein structure and splicing impairment. The paired sample feature from the DeNovoGear software was further used to examine potential de novo mutations in twin pairs.

Data analysis and filtering of mapped target sequences was performed with the 'Varbank' exome and genome analysis pipeline v.2.1 (unpublished; https://varbank.ccg.uni-koeln.de). In particular, we filtered for high-quality (coverage of more than six reads, fraction of allele carrying reads at least 25\%, a minimum genotype quality score of 10, VQSLOD greater than -8) and rare (Caucasian population allele frequency $<0.5 \%$ ) variations on targeted regions + flanking 100bp. In order to exclude pipeline specific artifacts, we also filtered against an in-house epilepsy cohort $(n=511, \mathrm{AF}<2 \%$ ) of variations, which were created with the same analysis pipeline. The filter conditions were set to be more sensitive following manual inspections of aligned reads. In particular, we looked for typical patterns of false positive variations (e.g., more than two haplotypes, base quality or mapping quality bias, strand bias, allele read position bias, low complexity region, alignment errors).

\section{Variation Analysis}

Variations identified by WES were amplified from DNA by polymerase chain reaction (PCR), and automated sequence analysis was carried out using standard procedures. In brief, primers were directed to all 13 variations observed and the resultant PCR products were subjected to direct automated BigDye Terminator sequencing (3130XL Genetic Analyzer, Applied Biosystems, Foster City, California, USA). Both strands from each amplicon were sequenced and presence of the variations in each twin pair was investigated by sequencing the respective PCR product.

\section{Results}

The only de novo variation identified in all twins by using the de novo probability tool DeNovoGear (c.233A $>$ G, p.Gln78Arg) was observed in TMPRSS13 (encoding transmembrane protein, serine 13) in one of the CHD-affected twins (twin pair 4). This variation had been detected in $0.9 \%$ of the alleles in 511 in-house epilepsy controls and was deposited in dbSNP Build144 (rs75037497). In the Exome Aggregation Consortium (ExAC), the allele frequency was highest for the Finnish (0.2\%) and East Asian (0.16\%) populations. However, Sanger sequencing did not confirm 


\section{TABLE 1}

Characterization of the Monozygotic Discordant Twins Investigated

\begin{tabular}{|c|c|c|c|c|}
\hline $\begin{array}{l}\text { Twin pair no. } \\
\text { and sex }\end{array}$ & Phenotype of the affected twin & Pregnancy & $\begin{array}{l}\text { Time of sample } \\
\text { recruitment }\end{array}$ & Siblings \\
\hline 1, male & L-transposition of the great vessels, ventricular septal defect & In term & After birth & No \\
\hline 2 , male & Ventricular septal defect & In term & After birth & No \\
\hline 3, male & Prolactinoma & - & At the age of 23 years & No \\
\hline 4, female & Hypoplastic left heart syndrome & - & At the age of 9 years & Yes, two \\
\hline 5 , male & Critical pulmonary valve stenosis & - & After birth & No \\
\hline 6 , female & Congenital diaphragmatic hernia & In term & After birth & No \\
\hline 7, female & $\begin{array}{l}\text { Total anomalous pulmonary venous connection, atrio-ventricular septal } \\
\text { defect, transposition of the great vessels }\end{array}$ & In term & After birth & No \\
\hline 8 , female & Omphalocele, partial hypoplasia of the corpus callosum. & In term & After birth & No \\
\hline 9 , female & Acromegaly & - & At the age of 40 years & No \\
\hline
\end{tabular}

this variation in the affected twin (see Supplementary figure for examples).

Analysis of all twins without DeNovoGear, using standard filter criteria (Table 2), detected 722 heterozygous variations that were only present in the affected twins and were absent in in-house controls. By comparing the read quality in Varbank with visual inspection, 12 variations remained for validation through Sanger sequencing. However, none of these variations could be confirmed. Either they were found to be false-positive calls in the affected twin or they turned out to be present in both twins.

\section{Discussion}

To our knowledge, this is the first WES study to search for genomic variations in discordant twins with $\mathrm{CHD}, \mathrm{CDH}$, omphalocele, prolactinoma, or acromegaly. Stringent analysis and comparison of WES data revealed no genetic differences between each of the discordant MZ twins.

Whereas a few studies have shown discordant twin phenotypes to result from dominant de novo mutations affecting coding regions, as described for Van der Woude syndrome (Kondo et al., 2002), our current findings suggest that such events are not a common cause for phenotypic discordance among MZ twins. This is in accordance with other studies in which no genetic differences in CNV profiles or coding regions were found between MZ twins discordant for several disorders including the following: (1) multiple sclerosis (Baranzini et al., 2010), (2) schizophrenia (Ono et al., 2010), (3) renal agenesis (Jin et al., 2014), (4) the VACTERL association (Solomon et al., 2013), (5) urogenital malformations (Baudisch et al., 2013), (6) caudal appendage with multiple congenital anomalies (Cogulu et al., 2013), (7) Crohn's disease (Petersen et al., 2014), (8) congenital hypothyroidism (Magne et al., 2015), (9) amyotrophic lateral sclerosis (Meltz Steinberg et al., 2015), or (10) congenital cataract (Wei et al., 2015). Abdellaoui et al. (2015) reported concordance rates of CNVs of $\sim 80 \%$ within 1,097 phenotypically unselected MZ twin pairs. However, only 2 of 20 putative de novo CNV candidates tested could be validated, suggesting that only a very small number of true post-twinning de novo CNVs may remain among this large number of MZ twin pairs. Moreover, whole-genome sequencing revealed that the genomes of 100-year-old MZ twins were discerned by only eight somatic single-base substitutions, whereas no variation was found in a 40 -year-old twin pair (Ye et al., 2013). Taking the estimate of Bruder et al. (2008), that de novo post-twinning CNV frequency could be as high as $5 \%$ on a per-individual basis or $10 \%$ per twinning event, all these data may rather support the assumption of an even more rare occurrence of post-twinning CNVs.

Several further explanations may account for our findings in the present study. First, WES covers only a small portion $(1-2 \%)$ of the genome and provides no information of non-coding mRNA elements and/or intronic or intergenic regulatory regions responsible for the discordant phenotypes of the respective twins. It is also known, that (1) a small fraction of exons are refractory to WES in current technologies and that (2) potential variations might be filtered out as a result of low coverage (Jamuar et al., 2014). Second, recent studies revealed substantial genetic variation in human tissue (O'Huallachain et al., 2012; 2013) and hence one may miss tissue-specific somatic mutations, especially in these tissues affected by the particular phenotype. Here, we investigated peripheral blood and testing of other tissue types that may have established a causative variation, as has been recently shown for Proteus syndrome (Lindhurst et al., 2011). Moreover, epigenetic differences may account for the disease, as reported by Kaminsky et al. (2009), who detected epigenetic variability between MZ twin pairs in all tissues tested. Supportive of this assumption, different DNA methylation, leading to dysregulation of gene expression, was found in MZ twins discordant for psoriasis or primary biliary cirrhosis (Gervin et al., 2012; Mitchell et al., 2011; Selmi et al., 2014). On the other hand, such changes could not be attributed to disease discordance in three multiple sclerosis discordant MZ twin pairs (Baranzini et al., 2010).

There are also technical limits of both next-generation sequencing (NGS) and BigDye/Sanger sequencing to detect low-frequency variants. For NGS, aside from the methods of library creation, pooling, and enrichment strategies, as 
TABLE 2

Analysis of All Twins Using Standard Filter Criteria Without DeNovoGear

\begin{tabular}{|c|c|c|c|c|c|c|c|c|c|}
\hline \multirow[b]{2}{*}{ Filter step } & \multicolumn{9}{|c|}{ Number of variations } \\
\hline & Twin_01 & Twin_02 & Twin_03 & Twin_04 & Twin_05 & Twin_06 & Twin_07 & Twin_08 & Twin_09 \\
\hline $\begin{array}{l}\text { Standard filter criteria in Varbank } \\
\text { Minimal read coverage: } 6\end{array}$ & 645 & 665 & 737 & 829 & 818 & 738 & 733 & 831 & 661 \\
\hline $\begin{array}{l}\text { Minimal variation quality: } 10 \\
\text { Affected twin with variation versus twin } \\
\quad \text { without variation }\end{array}$ & 124 & 113 & 179 & 124 & 175 & 115 & 133 & 189 & 118 \\
\hline Absence in in-house controls & 73 & 59 & 107 & 76 & 101 & 58 & 71 & 106 & 71 \\
\hline Visual inspection & 5 & 6 & 4 & 3 & 15 & 10 & 11 & 7 & 11 \\
\hline $\begin{array}{l}\text { Criteria of expression (OMIM, ZFIN, } \\
\text { MGI) }\end{array}$ & 5 & 2 & 1 & $1^{*}$ & 1 & 0 & 1 & 1 & 1 \\
\hline
\end{tabular}

well as the variant caller program, the coverage is highly important. Spencer et al. (2014) demonstrated that $>500 \mathrm{x}$ coverage is required for optimal performance for detecting low-frequency single nucleotide variants, hence this very expensive strategy is not performed on a regular basis. These authors reported that an analytical sensitivity of $>90 \%$ can be achieved when analyzing variant allele fractions of $10 \%$ by generating this high coverage. For routine Sanger sequencing, Tsiatis et al. (2010) found a limit of correct detection of $15-20 \%$ when testing tumor samples for the detection of KRAS mutations.

Taken together, the data of others and our study suggest that CNVs or exome DNA differences are very rare and that these might have a minor role in the pathophysiology of the complex diseases investigated. Hence, it is possible that the causes of the discordance among the presently investigated twin pairs may not be directly gene related but due to currently unidentified epigenetic or environmental factors. As outlined by Manolio et al. (2009), post-twinning de novo mutations will not contribute to family resemblance and heritability, but can explain some of the variation at present attributed to 'environment'. Since we could not confirm post-zygotic disease-causing mutations in any of the affected twins of the analyzed MZ discordant twin pairs, we have to assume that in an ACE $(\mathrm{A}=$ additive genetic factors, $C=$ shared environment effects, $E=$ unshared environment effects) twin model (Eaves et al., 1978), E plays a much more important role in their phenotypic discordance than A. This would concur with a low heritability of the present disease/birth defect in the offspring of the affected twin.

A contemporary approach to identifying post-zygotic variation explaining at least some of the 'missing' genetic contribution might be the expansion of sample sizes for MZ discordant twin-pairs in these disorders.

\section{Conclusions}

Genomic mutations in coding regions do not seem to play a major role in the discordance of MZ twins, although our cohort represents only a small number of MZ discordant twins. We cannot exclude that causative mutations may reside outside the regions accessible by WES or that discordance is the result of different epigenetic modifications.

\section{Acknowledgments}

We thank all families and patients for their participation in the study.

\section{Supplementary Material}

To view supplementary material for this article, please visit http://dx.doi.org/10.1017/thg.2015.93.

\section{References}

Abdellaoui, A., Ehli, E. A., Hottenga, J.-J., Weber, Z., Mbarek, H., Willemsen, G., ... Boomsma, D. I. (2015). CNV concordance in 1,097 MZ twin pairs. Twin Research and Human Genetics, 18, 1-12.

Baranzini, S. E., Mudge, J., van Velkinburgh, J. C., Khankhanian, P., Khrebtukova, I., Miller, N. A., ... Kingsmore, S. F. (2010). Genome, epigenome and RNA sequences of monozygotic twins discordant for multiple sclerosis. Nature, 464, 1351-1356.

Baudisch, F., Draaken, M., Bartels, E., Schmiedeke, E., Bagci, S., Bartmann, P., ... Reutter, H. (2013). CNV analysis in monozygotic twin pairs discordant for urorectal malformations. Twin Research and Human Genetics, 16, 802-807.

Breckpot, J., Thienpont, B., Gewillig, M., Allegaert, K., Vermeesch, J. R., \& Devriendt, K. (2012). Differences in copy number variation between discordant monozygotic twins as a model for exploring chromosomal mosaicism in congenital heart defects. Molecular Syndromology, 2, 81-87.

Bruder, C. E. G., Piotrowski, A., Gijsbers, A. A. C. J., Andersson, R., Erickson, S., Diaz de Ståhl, T., ... Dumanski, J.P. (2008). Phenotypically concordant and discordant monozygotic twins display different DNA copynumber-variation profiles. The American Journal of Human Genetics, 82, 763-771.

Cogulu, O., Pariltay, E., Koroglu, O. A., Aykut, A., Ozyurek, R., Levent, E., ... Ozkinay, F. (2013). Genome wide analysis 
in a discordant monozygotic twin with caudal appendage and multiple congenital anomalies. Genetic Counseling, 24, 85-91.

Dal, G. M., Ergüner, B., Sağiroğlu, M. S., Yüksel, B., Onat, O. E., Alkan, C., \& Ozçelik, T. (2014). Early postzygotic mutations contribute to de novo variation in a healthy monozygotic twin pair. Journal of Medical Genetics, 51, 455459.

Eaves, L. J., Last, K. A., Young, P. A. \& Martin, N. G. (1978). Model-fitting approaches to the analysis of human behavior. Heredity, 41, 249-320.

Ehli, E. A., Abdellaoui, A., Hu, Y., Hottenga, J. J., Kattenberg, M., van Beijsterveldt, T., ... Davies, G. E. (2012). De novo and inherited CNVs in MZ twin pairs selected for discordance and concordance on attention problems. European Journal of Human Genetics, 20, 1037-1043.

Gervin, K., Vigeland, M. D., Mattingsdal, M., Hammerø, M., Nygård, H., Olsen, A. O., ... Lyle, R. (2012). DNA methylation and gene expression changes in monozygotic twins discordant for psoriasis: Identification of epigenetically dysregulated genes. PLoS Genetics, 8, e1002454.

Helderman-van den Enden, A. T., Maaswinkel-Mooij, P. D., Hoogendoorn, E., Willemsen, R., Maat-Kievit, J. A., Losekoot, M., \& Oostra, B. A. (1999). Monozygotic twin brothers with the fragile X syndrome: Different CGG repeats and different mental capacities. Journal of Medical Genetics, 36, 253-257.

Jamuar, S. S., Lam, A. N., Kircher, M., D’Gama, A. M., Wang, J., Barry, B. J., ... Walsh, C. A. (2014). Somatic mutations in cerebral cortical malformations. New England Journal of Medicine, 371, 733-743.

Jin, M., Zhu, S., Hu, P., Liu, D., Li, Q., Li, Z., ... Chen, X. (2014). Genomic and epigenomic analyses of monozygotic twins discordant for congenital renal agenesis. American Journal of Kidney Diseases, 64, 119-122.

Kaminsky, Z. A., Tang, T., Wang, S.-C., Ptak, C., Oh, G. H. T., Wong, A. H. C., ... Petronis, A. (2009). DNA methylation profiles in monozygotic and dizygotic twins. Nature Genetics, 41, 240-245.

Kondo, S., Schutte, B. C., Richardson, R. J., Bjork, B. C., Knight, A. S., Watanabe, Y., ... Gershwin, M. E. (2002). Mutations in IRF6 cause Van der Woude and popliteal pterygium syndromes. Nature Genetics, 32, 285-289.

Kruyer, H., Mila, M., Glover, G., Carbonell, P., Ballesta, F., \& Estivill, X. (1994). Fragile X syndrome and the (CGG)n mutation: Two families with discordant MZ twins. American Journal of Human Genetics, 54, 437-442.

Lindhurst, M. J., Sapp, J. C., Teer, J. K., Johnston, J. J., Finn, E. M., .. Biesecker, L. G. (2011). A mosaic activating mutation in $A K T 1$ associated with the proteus syndrome. New England Journal of Medicine, 365, 611-619.

Magne, F., Serpa, R., Van Vliet, G., Samuels, M. E., \& Deladoëy, J. (2015). Somatic mutations are not observed by exome sequencing of lymphocyte DNA from monozygotic twins discordant for congenital hypothyroidism due to thyroid dysgenesis. Hormone Research in Paediatrics, 83, 79-85.

Manolio, T. A., Collins, F. S., Cox, N. J., Goldstein, D. B., Hindorff, L. A., Hunter, D. J., ... Visscher, P. M. (2009).
Finding the missing heritability of complex diseases. $\mathrm{Na}$ ture, 461, 747-753.

Meltz Steinberg, K., Nicholas, T. J., Koboldt, D. C., Yu, B., Mardis, E., \& Pamphlett, R. (2015). Whole genome analyses reveal no pathogenetic single nucleotide or structural differences between monozygotic twins discordant for amyotrophic lateral sclerosis. Amyotrophic Lateral Sclerosis and Frontotemporal, 16, 385-392.

Mitchell, M. M., Lleo, A., Zammataro, L., Mayo, M. J., Invernizzi, P., Bach, N., ... LaSalle, J. M. (2011). Epigenetic investigation of variably $\mathrm{X}$ chromosome inactivated genes in monozygotic female twins discordant for primary biliary cirrhosis. Epigenetics, 6, 95-102.

O’Huallachain, M., Karczewski, K. J., Weissman, S. M., Urban, A. E., \& Snyder, M. P. (2012). Extensive genetic variation in somatic human tissues. Proceedings of the National Academy of Sciences, USA, 109, 18018-18023.

O’Huallachain, M., Weissman, S. M., \& Snyder, M. P. (2013). The variable somatic genome. Cell Cycle, 12, 5-6.

Ono, O., Imamura, A., Tasaki, S., Kurotaki, N., Ozawa, H., Yoshiura, K., \& Okazaki, Y. (2010). Failure to confirm CNVs as of aetiological significance in twin pairs discordant for schizophrenia. Twin Research and Human Genetics, 13, 455460.

Petersen, B. S., Spehlmann, M. E., Raedler, A., Stade, B., Thomsen, I., Rabionet, R., ... Franke, A. (2014). Whole genome and exome sequencing of monozygotic twins discordant for Crohn's disease. BMC Genomics, 15, 564.

Selmi, C., Cavaciocchi, F., Lleo, A., Cheroni, C., De Francesco, R., Lombardi, S. A., ... Gerrshwin, M. E. (2014). Genomewide analysis of DNA methylation, copy number variation, and gene expression in monozygotic twins discordant for primary biliary cirrhosis. Frontiers in Immunology, 5, 128.

Solomon, B. D., Pineda-Alvarez, D. E., Hadley, D. W., Hansen, N. F., Kamat, A., Donovan, F. X., \& NISC Comparative Sequencing Program. (2013). Exome sequencing and highdensity microarray testing in monozygotic twin pairs discordant for features of VACTERL association. Molecular Syndromology, 4, 27-31.

Spencer, D. H., Tyagi, M., Vallania, F., Bredemeyer, A. J., Pfeifer, J. D., Mitra, R. D. \& Duncavage, E. J. (2014). Performance of common analysis methods for detecting low-frequency single nucleotide variants in targeted next-generation sequence data. The Journal of Molecular Diagnostics, 16, 7588.

Taylor, D. M., Thum, M. Y., \& Abdalla, H. (2008). Dichorionic triamniotic triplet pregnancy with monozygotic twins discordant for trisomy 13 after preimplantation genetic screening: Case report. Fertility and Sterility, 90, 2017, e5-e9.

Tsiatis, A. C., Norris-Kirby, A., Rich, R. G., Hafez, M. J., Gocke, C. D., Eshleman, J. R., \& Murphy, K. M. (2010). Comparison of Sanger sequencing, pyrosequencing, and melting curve analysis for the detection of KRAS mutations. Diagnostic and clinical implications. The Journal of Molecular Diagnostics, 12, 425-432.

Van Dongen, J., Slagboom, P. E., Draisma, H. H. M., Martin, N. G., \& Boomsma, D. I. (2012). The continuing value of 
twin studies in the omics era. Nature Reviews Genetics, 13, 640-653.

Wei, T., Sun, H., Hu, B., Yang, J., Qiao, C., \& Yan, M. (2015). Exome sequencing and epigenetic analysis of twins who are discordant for congenital cataract. Twin Research and Human Genetics, 18, 393-398.

Ye, K., Beekman, M., Lameijer, E.-W., Zhang, Y., Moed, M. H., van den Akker, E. B., ... Slagboom, P. E. (2013). Aging as accelerated accumulation of so- matic variants: Whole-genome sequencing of centenarian and middle-aged monozygotic twin pairs. Twin Research and Human Genetics, 16, 10261032.

Zwijnenburg, P. J., Meijers-Heijboer, H., \& Boomsma, D. I. (2010). Identical but not the same: The value of discordant monozygotic twins in genetic research. American Journal of Medical Genetics B Neuropsychiatric Genetics, 153B, 11341149. 